\title{
Conectando con nuestros abuelos para mejorar la educación formal Connecting with our grandparents to improve formal education
}

\author{
Silverio Alarcón ${ }^{1}$, Emilio Pindado ${ }^{1}$ \\ silverio.alarcon@upm.es, emilio.pindado@upm.es \\ ${ }^{1}$ Dept. Economía Agraria, Estadística y Gestión de Empresas \\ Universidad Politécnica de Madrid \\ Madrid, España
}

\begin{abstract}
Resumen- Esta experiencia surgió al inicio del curso 2020-21, al detectar la necesidad de fortalecer relaciones humanas tras meses de confinamiento por la COVID-19. Esta circunstancia se aprovechó para motivar a los alumnos en su proceso de aprendizaje. En concreto, el objetivo era aprender y aplicar técnicas de investigación social afrontando problemas de situaciones reales y próximas, en las que los alumnos pudieran desarrollar relaciones de empatía. Partiendo de unos conocimientos teóricos sobre investigación cualitativa, se propuso a 57 estudiantes, matriculados en una asignatura de Investigación de Mercados, realizar entrevistas en profundidad a sus abuelos para tratar los problemas surgidos durante el periodo de confinamiento, con especial referencia a cuestiones de habitabilidad. Se obtuvieron treinta transcripciones que se usaron para realizar el trabajo de la asignatura. Los resultados muestran que la experiencia ha sido enriquecedora para los estudiantes en cuanto que ha permitido aplicar métodos del programa de la asignatura a casos reales, cercanos, y con interés social. De forma más general, se propone potenciar el contacto con los abuelos de los alumnos, dada su proximidad y sus conocimientos, para impulsar la implicación de la enseñanza universitaria en los Objetivos de Desarrollo Sostenible.
\end{abstract}

\section{Palabras clave: investigación cualitativa, aprendizaje informal, COVID-19, habitabilidad.}

\begin{abstract}
This experience arose at the beginning of the 2020-21 academic year, when detecting the need to strengthen human relationships after months of confinement by COVID-19. This circumstance was used to motivate the students in their learning process. Specifically, the objective was to learn and to apply social research techniques facing problems in real and close situations, in which students could develop empathic relationships. Based on theoretical knowledge on qualitative research, 57 students, enrolled in a Market Research course, were asked to carry out in-depth interviews with their grandparents to deal with the problems that arose during the confinement period, with special reference to habitability issues.. Thirty transcripts were obtained and used to carry out the work of the subject. The results show that the experience has been enriching for the students insofar as it has allowed to apply the methods of the subject program to real cases, close, and with social interest. More generally, it is proposed to promote contact with the students' grandparents, given their proximity and their knowledge, to promote the involvement of university education in the Sustainable Development Goals.
\end{abstract}

Keywords: qualitative research, informal learning, COVID-19, habitability.

\section{INTRODUCCIÓN}

La enseñanza formal puede enriquecerse si aprovechamos la enseñanza informal que nos puede proporcionar nuestro entorno. Por una parte, el mundo más cercano en el que vivimos (la familia, los amigos, el barrio, la universidad, etc.) está repleto de oportunidades y temas de interés para estudiar en el aula (Alarcón y López-Cózar, 2018; López-Cózar et al., 2019). Por otra, la enseñanza informal que tradicionalmente proporcionaban las personas mayores a los más jóvenes se ha perdido en las sociedades modernas, y ha sido sustituida por la enseñanza formal que se imparte en las instituciones de educación (Dewey, 1923). Sin embargo, el proceso de aprendizaje se puede mejorar si recuperamos estas enseñanzas de nuestros mayores, y las usamos para centrar trabajos prácticos de las asignaturas. De esta forma se atenúan los riesgos derivados de la división entre la experiencia que proporciona el aprendizaje informal y el que se adquiere en la universidad (Dewey, 1923).

Adicionalmente a este planteamiento, el periodo de confinamiento, marzo a junio de 2020 , originó en la población situaciones de soledad o disminución de comunicación entre familiares que súbitamente se encontraron separados. Reestablecer estas conexiones y contar las experiencias vividas es en sí algo positivo, pero además se puede usar como recurso para mejorar la calidad de la educación (Martínez, 2017; Centeno et al., 2019; Alonso et al., 2020).

Este trabajo se centra precisamente en proponer a los alumnos que realicen entrevistas en profundidad a sus abuelos para que les cuenten las experiencias vividas en este periodo. Creemos que la comunicación con los abuelos es una oportunidad para que los alumnos apliquen mejor los conocimientos teóricos a casos reales y cercanos, y de paso fortalezcan lazos y aprendan algo de ellos. No se pone el énfasis en que los alumnos desarrollen soluciones innovadoras, sino más bien en que se sumerjan en las complejidades de los problemas sociales, y en que los lazos afectivos contribuyan al proceso de aprendizaje (Eyler y Giles, 1999). No se pretende realizar aprendizaje-servicio a este colectivo. En la clasificación de Sigmon (1996) se podría considerar próxima a APRENDIZAJE-servicio, es decir el aprendizaje es el objetivo principal mientras que el servicio es secundario. En cualquier caso, el servicio no lo proporcionan los alumnos sino los 
abuelos, o los actores entrevistados, de los que se espera obtener algún tipo de conocimiento o enseñanza informal. Por este motivo, se propone la denominación aprendizaje-servicioinverso.

Los resultados muestran que efectivamente esta propuesta fue una referencia útil en distintas etapas del desarrollo de la asignatura y en la adquisición de competencias y conocimientos prácticos, y fue valorada positivamente por los alumnos.

El siguiente apartado trata el contexto en el que se desarrolla la asignatura, las necesidades detectadas, las oportunidades de mejora que se han identificado y los objetivos, tanto los que se propusieron a los alumnos como los de esta experiencia docente. En la sección 3 se describen los detalles de las actividades que se desarrollaron y en la 4 se muestran los resultados y las evaluaciones respecto a varios criterios. Por último, las conclusiones hablan de limitaciones, aspectos a mejorar en futuras experiencias y la transferibilidad a otras asignaturas.

\section{CONTEXTO}

La experiencia se enmarca en la asignatura Investigación de Mercados que pertenece al plan de estudios del doble grado en Edificación y Administración de Empresas de la Universidad Politécnica de Madrid. Es, por tanto, una asignatura de perfil socioeconómico en unos estudios universitarios con contenidos principalmente técnicos.

En esta asignatura se imparten métodos cualitativos y cuantitativos para explorar los gustos y necesidades de los consumidores. Es obligatorio realizar un trabajo de la asignatura que consiste en un estudio de mercado o social sobre un bien o servicio relacionado con la titulación. No necesariamente tiene que ser un producto comercial. Se permite abordar trabajos empíricos sobre cuestiones sociales, siempre que tengan alguna conexión con las enseñanzas que se imparten en la Escuela Técnica Superior de Edificación. Los trabajos son predominantemente de tipo cuantitativo y los alumnos desarrollan las etapas de planificar y diseñar un cuestionario, lanzar la encuesta a la población objetivo, capturar, depurar y analizar estadísticamente la información, y elaborar un informe que presentan en público.

En raras ocasiones los alumnos se deciden por abordar trabajos de tipo cualitativo. Probablemente se deba a que su formación matemática y estadística les incline hacía estudios cuantitativos en los que se desenvuelven más cómodamente. De aquí surge la necesidad de reforzar los métodos cualitativos de la asignatura y de generar material por parte de los alumnos (por ejemplo, transcripciones de entrevistas en profundidad) para que sea posible acometer trabajos aplicados cualitativos. Además de esto, y quizá más importante y general, existe una necesidad de conseguir que los conocimientos teóricos se asimilen con aplicaciones prácticas reales que están al alcance de los alumnos.

La pandemia de COVID-19 ha ocasionado muchos fallecidos en todo el mundo y ha deteriorado la situación económica y las condiciones de vida de muchas familias. Paradójicamente también ha creado muchas oportunidades de investigación en todos los campos, como se puede comprobar en cualquier revista científica o de divulgación. También la educación puede mirar a este periodo para encontrar oportunidades de innovación educativa que nos ayuden a mejorar la docencia. En concreto, el confinamiento de marzo a junio 2020 ha sido el motivo para realizar entrevistas en profundidad entre colectivos que probablemente hayan estado aislados o con poca comunicación durante este periodo. El objetivo de las entrevistas en profundidad era hablar de los problemas de habitabilidad que habían tenido las personas mayores durante el confinamiento, y de forma más general de cómo habían vivido esos tres meses. Los objetivos de esta experiencia educativa eran: (1) aplicar los conocimientos de la técnica de entrevista en profundidad a un caso real y cercano real a los alumnos e iniciar a los alumnos como entrevistadores; (2) motivar a los alumnos, implicar a sus abuelos y reforzar lazos tras el confinamiento.

\section{DESCRIPCIÓN}

La experiencia tuvo 3 fases: diseño de un guion, realización de entrevistas, y elaboración de informes.

Diseño de un guion. Después de impartir conocimientos teóricos sobre métodos cualitativos y sobre la técnica de entrevista en profundidad (EP), se programó una tarea en la que los alumnos debían elaborar un guion EP para abordar el tema de los problemas de habitabilidad que habían tenido los entrevistados durante el confinamiento marzo-junio de 2020. No se fijó la población objetivo en este momento, solamente se sobreentendía que las EPs se realizarían a adultos. Es más, se preguntaba sobre qué colectivos sería más interesante realizar esta EP. La intención era que los alumnos se centraran en cómo diseñar una EP con capacidad para obtener experiencias humanas surgidas en situaciones no habituales. Esta tarea fue individual $y$ tuvieron una semana para realizarla. Posteriormente en clase, se mostraron y discutieron todos los guiones, y se eligió uno común, que es el que utilizaron en la siguiente fase.

Realización de entrevistas. En este momento es cuando se fijó la población objetivo. Se decidió que fueran los abuelos de los alumnos, porque el aislamiento de COVID-19 había sido especialmente duro con las personas mayores, y por tanto había más interés social en identificar las experiencias vividas por estas personas durante el confinamiento e identificar los problemas de habitabilidad que hubieran podido tener. La tarea fue también individual y disponían de 2 semanas. Cada alumno debía realizar al menos una EP a uno de sus abuelos, independientemente de su edad y de su lugar de residencia, conforme al guion común y siguiendo las indicaciones. Las EPs podían ser presenciales $\mathrm{u}$ online, y como resultado debía entregarse una transcripción en formato pdf, anónima de entrevistador y entrevistado. Al final de la transcripción se pedía que el entrevistador expresara su opinión sobre los comentarios del entrevistado. El texto de esta tarea se muestra en el Anexo.

Elaboración de informes. Todas las transcripciones se empaquetaron en un archivo comprimido y se puso a disposición de todos los alumnos para que las usaran voluntariamente en el trabajo de la asignatura. En este caso, la actividad era en grupo y dispusieron de 2 meses para realizarla, aunque se programó una entrega parcial con el procesamiento de la información.

\section{RESUltados}

En la primera fase, los alumnos tuvieron la oportunidad de aplicar los conocimientos teóricos sobre métodos cualitativos 
impartidos en clase a una situación real. En esta aplicación aprovecharon sus conocimientos técnicos sobre edificación y condiciones de habitabilidad, y su propia experiencia durante el confinamiento, para identificar puntos de interés a tratar en la EP. Fue una actividad sencilla y motivadora, en la que participó una parte importante de los alumnos $(45 / 58,77,6 \%)$, y permitió que visualizaran los problemas que surgen al pasar de la teoría a la práctica. El impacto fue desigual entre los alumnos, pues unos se lo tomaron más en serio y otros no tanto. Los profesores de la asignatura valoramos positivamente que se obtuvieron al menos 4 ó 5 guiones de calidad, que permitieron un debate de altura en la clase posterior, y llevó a consensuar un guion definitivo. El principal inconveniente que se detectó es que los alumnos percibieron esta actividad como una tarea rutinaria más de la asignatura. Es decir, a pesar de que se eligió un tema de actualidad relacionado con sus conocimientos técnicos, no se encontró la motivación que se esperaba.

El texto consensuado del guion se muestra en el Anexo. Puede verse que en este guion coexisten cuestiones habituales rutinarias, personales, sociales y técnicas de la vivienda.

En la segunda actividad, realización de entrevistas, la participación fue menor $(31 / 58,53,4 \%)$, pero aumentó la proporción de aportaciones (transcripciones) de calidad. De los 31 alumnos que realizaron EP, 12 obtuvieron una calificación de sobresaliente, 16 notable y 3 aprobado. Además, en este caso, sí se puede afirmar que los alumnos valoraron positivamente entrevistar a sus abuelos para recabar su opinión sobre cuestiones de experiencias propias en relación con sus estudios. Los comentarios más frecuentes, en las valoraciones de las EPs, son los que muestran aspectos personales o afectivos que reflejan sentimientos de pena, soledad, alegría por el reencuentro o esperanza:

"La entrevista se ha realizado a través de una llamada de teléfono en la cual no se ha apreciado los gestos ni las expresiones del entrevistado, pero sí podemos observar cuando le preguntamos si habian pasado algún momento malo por el tiemblo en las palabras de tristeza al contarnos la muerte de sus amigos y el sentimiento de frustración al no poder hacer nada ante ello ni poder verles ni estar con ellos. Por lo demás la entrevista ha sido rápida fluida y por último cabe destacar lo importante que es hablar con los abuelos que nunca sabes cuando no los vas a tener." EP4

“Juan de 70 años nos ha mostrado su lado más personal y cómo ha llevado el confinamiento día a día. Le hemos visto pasarlo mal al recordar a amigos que ya no están con nosotros y como sufría por la situación llegando incluso a tener ansiedad de no poder salir y estar con los suyos. Pero a día de hoy le vemos más contento ya que por fin, aunque no como antes, puede estar con sus nietos ya que para él ellos son lo más importante de su vida." EP5

"En conclusión, tenemos que cuidar mucho a nuestros mayores porque son los más vulnerables y estar siempre disponibles para ellos, porque se avecinan dias duros de nuevo y no podemos dejar que se sientan tan solos." EP8

“Así termina la entrevista a Elena. Estuvo muy abierta a todas las preguntas y feliz de participar. Ella misma decía que ha tenido suerte. No lo ha pasado bien, pero 'podría haber sido peor'...Ha pasado mucho tiempo sola, pero estando acostumbrada no le ha costado tanto como podría esperarse. Por supuesto, está cansada y se nota un desgaste grande por esta situación. No es la misma Elena que cuando comenzó el confinamiento y ella se lo nota, se siente mayor de lo que se sentía antes." EP12

"En conclusión, el sujeto entrevistado no mostró ninguna inconformidad en cuanto a su vivienda. Por otro lado, se pude apreciar una gran tristeza al expresar su soledad y el hecho de no poder ver a su familia, que en mi opinión es el gran problema para las personas de estas edades.” EP18

" $Y$ en mi opinión espero que este tipo de entrevista sirva para concienciar a la gente de lo mal que lo pasan las personas mayores, y personas de riesgo y asi unirnos todos y comportarnos adecuadamente y si es asi venceremos al virus cuanto antes." EP23

"Para concluir, señalaré que en general la entrevista ha transcurrido de una forma positiva y tranquila. Al tratarse de una entrevista telefónica no me ha permitido llegar a percibir algunas reacciones de las que nos podríamos dar cuenta en persona, teniendo a la persona entrevistada delante nuestra, pero he podido notar el cambio en el tono de voz por algunas cuestiones. Por ejemplo, la angustia que transmitía mientras contaba sobre cómo ha visto la situación del confinamiento y como de preocupada estaba por su salud y la de los seres queridos. Sin duda, ha sido una experiencia que me ha resultado satisfactoria, ya que nunca viene mal escuchar a nuestros mayores y mantener la cercanía con ellos en todos los temas que nos afectan.” EP25

En menor medida hay comentarios neutros que inciden en que la EP les ha permitido comprender mejor la situación vivida por la persona mayor. Por ejemplo:

"Ha transcurrido de manera muy natural y sin ningún tipo de impedimentos. La persona entrevistada estaba muy dispuesta a contestar todo aquello que se le propusiese, lo cual ha facilitado la tarea. Sus expresiones me han ayudado a confirmar todo aquello que respondia." EP1

"Una vez terminada la entrevista, como conclusión saco que el mayor problema que pueden haber sufrido las personas que no han tenido inconvenientes con su vivienda, ha sido el estar encerrados en casa, y más aún si viven solos." EP11

"Durante toda la entrevista, he presenciado al entrevistado bastante calmado, pero si que se le podía apreciar un poco disgustado con toda la situación que nos está tocando vivir. A pesar de eso, se le ve con optimismo, positivismo y con ganas de salir adelante y avanzar." EP26

Y también son poco frecuentes son los que se centran en cuestiones técnicas de las viviendas o sus soluciones. Por ejemplo:

"Admite arrepentirse de haber cerrado la terraza del salón, le gustaría volver a abrirla pero no le compensa meterse en una obra ahora." EP8

"Después de este confinamiento y opiniones como las de Ángel, es de vital importancia tener una buena iluminación y a poder ser un pequeño balcón en las casas para poder estar más a gusto." EP14

"El tipo de vivienda y su acondicionamiento ha sido un factor determinante para llevar el confinamiento de la mejor manera posible, haciendo mucho más complicada la convivencia en zonas menos favorecidas, como también menciona en la entrevista." EP15 
La Figura 1 muestra el análisis de sentimientos realizado sobre las valoraciones de los alumnos, utilizando el paquete syuzhet del software R (Jockers, 2017). Este análisis refuerza los resultados anteriores, al ser los sentimientos más frecuentes la tristeza y el miedo, seguido de la confianza.

Conectando con nuestros abuelos para mejorar la educación formal

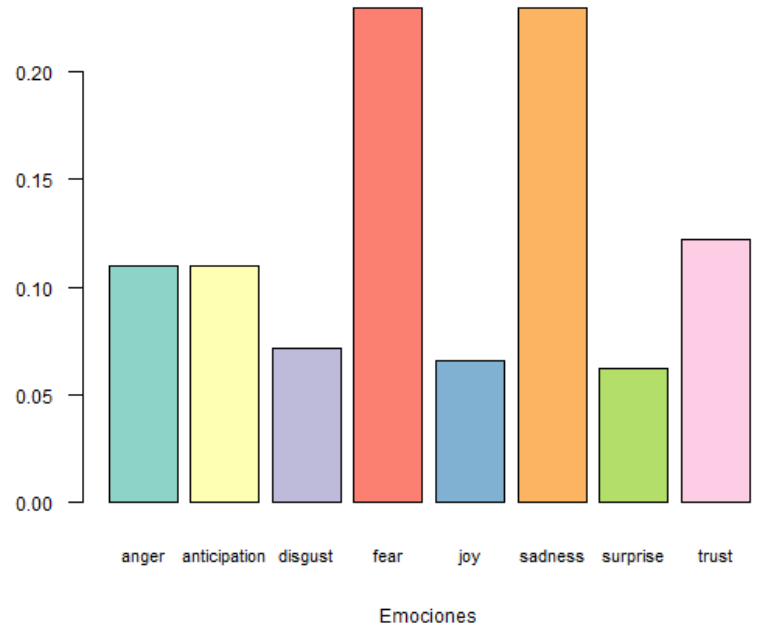

Figura 1. Análisis de sentimientos valoraciones alumnos.

En cualquier caso, estos comentarios indican que los alumnos en su función de entrevistadores comprendieron y adquirieron unas habilidades prácticas. Este aprendizaje es en sí la mayor aportación de esta experiencia educativa. Una cosa es explicar en clase la teoría sobre cómo se debe conducir una EP para mantenerse neutro y no influir sobre las opiniones del entrevistado, y otra es crear las condiciones para que los propios alumnos se enfrenten a estas situaciones y descubran cómo se han de desenvolver para conseguir los objetivos marcados. Evidentemente no todos los estudiantes se metieron en el papel de un buen entrevistador ni se preocuparon de desarrollar convenientemente sus habilidades, pero una buena parte de las transcripciones sí muestran un trabajo de calidad.

La tercera actividad fue procesar la información contenida en las 28 transcripciones (se descartaron las 3 con calificación de aprobado, por la escasa información que recogían) y elaborar un informe. El trabajo de la asignatura es obligatorio y supone el 30\% de la nota final, pero elegir esta opción es una decisión voluntaria. Solamente un grupo de tres alumnas tomó esta opción, y el resto se decantó por trabajos con métodos cuantitativos. No se puede hablar, por tanto, de un gran éxito. En cuanto a la calidad del trabajo, sí se puede decir que estuvo entre los tres mejores, con una calificación de sobresaliente, y los profesores valoramos el esfuerzo realizado por las alumnas para sintetizar toda la información contenida en las transcripciones. No obstante, si se compara con un trabajo profesional se percibe un tratamiento quizá demasiado mecánico y cierta carencia de contextualización. Creemos que estas deficiencias no son achacables a los alumnos y permiten identificar vías de mejora en los próximos cursos.

Resumimos muy brevemente los resultados del trabajo (López et al., 2020):

- Se realizaron más entrevistas a mujeres que a hombres.
- La mayoría de las personas entrevistadas residían en la Comunidad de Madrid (64,3\%).

- 16 entrevistados vivían en pisos y 11 en viviendas unifamiliares.

- $\quad$ Solamente un 28\% manifestaron haber tenido problemas de habitabilidad. Son frecuentes que estos problemas se den en personas que viven en pisos.

- Una parte importante de los entrevistados, casi la mitad, manifiesta haber tenido ánimo bajo como consecuencia del confinamiento, y de nuevo este estado ocurre en mayor medida entre los que viven en pisos. Se ven a sí mismos como el grupo de población más afectado por la pandemia.

- Algunos señalan la necesidad de contar con viviendas que dispongan de espacios abiertos.

\section{CONCLUSIONES}

Los comentarios de los alumnos muestran que la experiencia ha sido enriquecedora para ellos en cuanto que ha permitido aplicar métodos del programa de la asignatura a casos reales, cercanos, y con interés social. Destacamos como mayor aportación el papel de entrevistadores que han afrontado, y que contribuye a sus habilidades de comunicación y empatía. También creemos que ha sido positivo para los abuelos involucrarles en el proceso educativo de sus nietos, e intentar reforzar vías de comunicación que parecían necesarias en la pandemia.

Son varios los aspectos de esta experiencia que procuraremos mejorar en el próximo curso, en la asignatura Investigación de Mercados. En primer lugar, se pretende motivar más a los estudiantes en la fase de elaboración del guion de EP, o de otra técnica cualitativa. Una posibilidad es realizar una sesión de discusión para debatir los posibles temas y objetivos para la EP, en función de cuestiones o problemas de interés en relación con sus estudios o con su entorno. En segundo lugar, aunque los trabajos de la asignatura son tutelados y se realizan entregas parciales para que tengan un desarrollo progresivo y un resultado de calidad, se va a programar una mayor dedicación a explicar cómo elaborar informes de tipo cualitativo. Y en tercer lugar, en el próximo curso se introducirán encuestas que proporcionen indicadores cuantitativos para una evaluación más objetiva de esta experiencia educativa, tanto en lo que se refiere a los alumnos como a los abuelos.

En relación con la transferencia a otros contextos, creemos firmemente que este tipo de entrevistas a los abuelos pueden jugar un gran papel en las enseñanzas actuales. Reestablecer a nuestros abuelos a su función de educadores informales y transmisores de sus experiencias y vivencias es una fuente fructífera de conocimiento (Alonso et al., 2020). Tienen mucho que enseñarnos pues llevan toda una vida adquiriendo conocimientos, y tienen capacidad para aportar ideas y motivar la educación más formal que impartimos en las universidades. En estos momentos en los que los Objetivos de Desarrollo Sostenible (ODS) de Naciones Unidas son una referencia en todas las enseñanzas, los abuelos de nuestros estudiantes pueden aportar pistas de cómo avanzar hacia un planeta más justo y respetuoso con el medioambiente, pues son testigos de los cambios en la sociedad en las últimas décadas. Sus testimonios se pueden combinar con el conocimiento científico, social y técnico de las universidades para proponer soluciones. 


\section{AgRADECIMIENTOS}

Los autores agradecen a David Pérez y Ana Centeno por su presentación "El legado de los abuelos agricultores" en el V Congreso CINAIC de 2019, que ha sido fuente de reflexión para el desarrollo de esta experiencia educativa, así como a Ana Sánchez Calles y a dos revisores anónimos por sus comentarios a un primer borrador de esta comunicación, que han servido para mejorar considerablemente la versión final.

\section{REFERENCIAS}

Alarcón, S., López-Cózar, C. (2018). Retos en el entorno del estudiante como instrumento de motivación. I Jornadas de innovación docente en grados y posgrados en Ciencias Experimentales e Ingenierias, Septiembre, 2018, Universidad Rey Juan Carlos.

Alonso, R. A., Sáenz de Jubera, M., \& Sanz, E. (2020). Tiempos compartidos entre abuelos y nietos, tiempos de desarrollo personal. Revista Española de Pedagogía, 78(277), 415434.

Centeno, A., Pérez, D., Casas, R., Moratiel, R., \& Baeza, P. (2019). El legado de los abuelos agricultores. V Congreso Internacional sobre Aprendizaje, Innovación y Competitividad (CINAIC 2019), Octubre 9-11, 2019.

Dewey, J. (1923). Democracy and education: An introduction to the philosophy of education. Macmillan.

Eyler, J., \& Giles Jr, D. E. (1999). Where's the Learning in Service-Learning? Jossey-Bass Higher and Adult Education Series. Jossey-Bass, Inc., 350 Sansome St., San Francisco, CA 94104.

Jockers, M. (2017). Package 'syuzhet'. URL: https://cran. rproject. org/web/packages/syuzhet.

López, M. Maier, S.C. \& Porras, L. (2020). Trabajo final de Investigación de Mercados. Curso 2020-21. E.T.S. Edificación. Universidad Politécnica de Madrid. [no publicado].

López-Cózar, C., Alarcón, S., Corro, R. (2019). Análisis del interés por el emprendimiento social entre estudiantes universitarios. $V$ Congreso Internacional sobre Aprendizaje, Innovación y Competitividad (CINAIC 2019), Octubre 9-11, 2019.

Martínez, A. L. (2017). El rol de agentes educativos en los abuelos del siglo XXI: transmisión de valores $\mathrm{y}$ principales factores que influyen en el grado de relación mantenida con sus nietos. La Razón Histórica. Revista hispanoamericana de Historia de las Ideas, 37, 44-76.

Sigmon, R. L. (1996). Journey to Service-Learning: Experiences from Independent Liberal Arts Colleges and Universities. Council of Independent Colleges, Washington, DC.

\section{ANEXO}

El texto exacto de la tarea para elaborar las entrevistas en profundidad fue:

"El objetivo de esta tarea es realizar de forma individual una entrevista en profundidad semiestructurada sobre el tema:
Problemas de habitabilidad en viviendas como consecuencia de los confinamientos de COVID19.

La población objetivo son vuestros abuelos, es decir, personas mayores de más riesgo que son susceptibles de haber vivido mayores restricciones de espacio o peores condiciones en sus viviendas. Se puede realizar mediante contacto directo o por teléfono, videoconferencia o cualquier otro procedimiento. En estos últimos casos se debe mencionar en el documento.

Os adjunto el guion que tenéis que seguir (puede verse más adelante). Os recomiendo que iniciéis la entrevista con una breve introducción sobre el tema, y para ello ... os pueden servir de ayuda. A partir de ahí debéis dejar hablar libremente al entrevistado. Os recuerdo que no hay que controlar el orden ni los tiempos, y no hacer preguntas si no es necesario. Solamente centrar el tema en caso de que el entrevistado se desvie, o plantear preguntas del guion en caso de que se quede callado. Más adelante podéis plantear aquellos temas del guion que no se hayan tratado y las preguntas básicas de aspectos sociodemográficos: edad, género, código postal y tipo de vivienda.

El documento que tenéis que entregar es un pdf que recoja la transcripción de la entrevista o un resumen de la misma, respetando siempre el orden en el que se trataron los temas en la entrevista (que no tiene que coincidir con el del guion). También debéis incluir la información de las cuestiones sociodemográficas, y vuestros comentarios y valoración. Sobre esto último os recuerdo que en una entrevista en profundidad el entrevistador puede aportar información obtenida mediante observación, es decir, comportamiento, reacciones, estados de ánimo, etc. del entrevistado.

Por último, el pdf no debe incluir identificación ni del entrevistado ni del entrevistador."

El texto final consensuado del guion fue:

\section{“ENTREVISTA EN PROFUNDIDAD: PROBLEMAS DE}

HABITABILIDAD. En esta entrevista se analizarán los principales problemas de habitabilidad que se han tenido que afrontar en las viviendas durante el periodo de confinamiento. Se realizará una entrevista semiestructurada en la que habrá unos temas a tratar y no se controlará el orden o el tiempo. Los principales puntos a tratar serán:

- Actividades desarrolladas en la vivienda durante el confinamiento. Se preguntará sobre la capacidad que han tenido los entrevistados de realizar actividades cotidianas dentro de la vivienda (trabajo, estudio, ocio, ejercicio físico...)

- Problemas causados por el confinamiento. Se hablará sobre el estado de ánimo de los entrevistados durante el confinamiento, la convivencia con los vecinos...

- Problemas estructurales de la propia vivienda. Dentro de este punto se hablará sobre temas de iluminación, insonorización, aislamiento térmico, estructuras de balcones y tamaño y distribución de la vivienda.

- Soluciones a esos problemas. Se tratará de encontrar las razones que han provocado los problemas durante el confinamiento y se buscarán soluciones para aplicar en reformas y nuevas construcciones." 